\title{
MMSE Based Greedy Eigenmode Selection for AF MIMO Relay Channels
}

\author{
Shenyu Song, and Wen Chen, \\ Department of Electronic Engineering \\ Shanghai Jiao Tong University \\ Shanghai, China 200240 \\ Email: \{chimera,wenchen\}@sjtu.edu.cn
}

\begin{abstract}
In this paper, we consider a dual-hop amplifyand-forward (AF) multiple-input multiple-output (MIMO) relay system, where there is one source, one destination and $K$ relay nodes. We propose a minimum mean squared error (MMSE)based eigenmode selection algorithm to choose several relay nodes to minimize MSE. We use eigenmode-based definitions for the equivalent backward and forward channels, and extend the present MMSE-based selection scheme to multiple power amplifier configuration. According to the closed form expression of MSE, we iteratively select eigenmode pairs to minimize the MSE. Simulation results show that our scheme outperforms the existing ones.
\end{abstract}

Index Terms-Eigenmode, relay selection, MIMO relay, MMSE.

\section{INTRODUCTION}

Cooperative relaying has proved to be an effective way to achieve performance gains in wireless communication systems, to mitigate wireless fading and improve quality of service (QoS). A key issue in cooperative relay communication is relay selection (RS) [1]-[4], which is a feasible way to improve system performance with lower complexity and power consumption. [1] provides a basic protocol for relay selection, a famous "max-min" selection criterion, which is widely used in many works in this area. [2] investigates several relay selection schemes and demonstrates their diversity orders, including single-RS and multiple-RS (according to the number of selected relays). [3] and [4] provide an adaptive relay selection mechanism, in which the selection process is "automatically" executed by relays, requiring no supervisor program at the destination node.

On the other hand, relay networks with multi-antenna configuration have recently received considerable attention [5]-[9]. [5] analyzes the capacity of a multi-antenna relay system. [6] provides some strategies, such as backward and forward zeroforcing, to improve the system capacity. [7]-[9] propose several relay/antenna selection criterions for MIMO relay channel. In [7], the author proposes a selection scheme based on harmonic mean of the dual-hop channel gains, to maximize the achievable rate and minimize the interference as well. In [8], an iterative antenna selection scheme is provided, which is based on semi-orthogonality among spatial eigenmode and antenna pairs of relays. [9] proposes a greedy antenna selection algorithm to minimize the MSE resulted from selecting an additional relay antenna.

However, most of the existing schemes are based on heuristic method, such as "max-min" scheme, harmonic-mean scheme, etc. Although [9] derives a closed form of MSE and proposes an algorithm based on it, the author supposes that only one antenna pair can be activated at each relay node (single power amplifier, SPA). In this paper, we propose an eigenmode selection scheme based on [9]. By using eigenmode-based definitions for the equivalent backward and forward channels, we extend the presented MMSE-based selection scheme to multiple power amplifier (MPA) configuration. Simulation results show that our scheme outperforms the existing ones.

The remainder of this paper is organized as follows: Section II describes the system model of a dual-hop AF MIMO relay network. Section III demonstrates the proposed algorithm. Section IV gives simulation results. Conclusions are made in Section V.

Notation: In this paper, $(\cdot)^{H}$ and $(\cdot)^{-1}$ denote the Hermitian and inverse form of a matrix (vector) respectively. $\operatorname{det}(\cdot)$ and $\operatorname{tr}(\cdot)$ are the determinant and trace of a matrix respectively. $(\mathbf{A})_{i, j}$ is the entry at $i$ th row and $j$ th column of matrix $\mathbf{A}$. $\mathbf{I}_{N}$ stands for an $\mathrm{N} \times \mathrm{N}$ identity matrix. $\mathbf{0}^{M \times N}$ represents an $\mathrm{M} \times \mathrm{N}$ zero matrix. $\mathbb{E}\{\cdot\}$ is the expectation of a random variable. $A \triangleq B$ means that $\mathrm{A}$ is defined as $\mathrm{B}$.

\section{System Model}

We consider a MIMO relay system with one source $(S)$, one destination $(D)$ and $K$ relays $\left(R_{1}, \ldots, R_{K}\right)$ as illustrated in Fig. 1, where the nodes are equipped with $N_{S}, N_{D}$ and $N_{R}$ antennas respectively. For simplicity and practical consideration, a non-regenerative and half duplex relaying strategy is applied at the relay nodes to amplify and forward the received signals. The first time slot is assigned to the backward transmission, while the second one to the forward. $\mathbf{H}_{k} \in \mathbb{C}^{N_{R} \times N_{S}}$ and $\mathbf{G}_{k} \in \mathbb{C}^{N_{D} \times N_{R}}(k=1, \ldots, K)$ denote the $k$ th backward and forward channel coefficients respectively.

We assume that the destination does not receive any signal directly from the source. All the channel coefficients are independent identically distributed (i. i. d) random variable, and suppose that all the channels are frequency-flat block fading. 


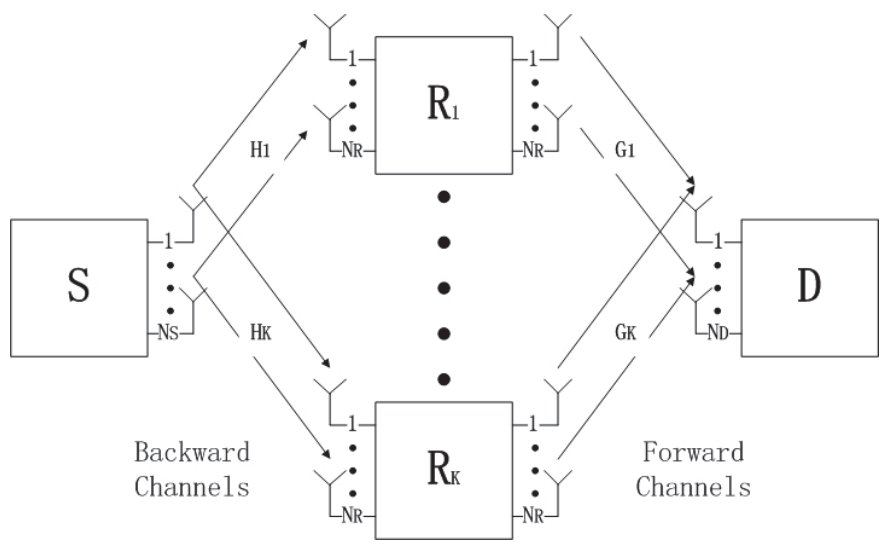

Fig. 1. Model of a MIMO relay channel.

In this paper, we consider the case with $K \geq \max \left\{N_{S}, N_{R}\right\}$. Therefore, the multiplexing gain of this system is limited by $M=\min \left\{N_{S}, N_{R}\right\}$. In order to obtain full multiplexing gain, the quantity of selected relays should exceed or at least be equal to $M$, as we regard the link $S \rightarrow D$ through the relays as a compound MIMO link. In [7] [8], the number of selected relays is set to $M$. In our paper, we will select more than $M$ relays to exploit more diversity gain.

We select $L$ relays $R_{r_{1}}, \ldots, R_{r_{L}}$ in the system to retransmit the signal from $S$ to $D$. During the first time slot, $S$ transmits a signal vector $\mathbf{s} \in \mathbb{C}^{N_{S} \times 1}$ to relay nodes. Let $P_{S}$ denote the transmit power constraint at source node,

$$
\mathbb{E}\left\{\operatorname{tr}\left(\mathbf{s s}^{H}\right)\right\} \leq P_{S}
$$

We assume equal power allocation for the transmitted data with full power. Hence, $\mathbb{E}\left\{\mathbf{s s}^{H}\right\}=\sigma_{s}^{2} \mathbf{I}_{N_{S}}$. We can write the received signal at relay $R_{r_{\ell}}(\ell=1, \ldots, L)$ as:

$$
\mathbf{y}_{r_{\ell}}=\mathbf{H}_{r_{\ell}} \mathbf{s}+\mathbf{n}_{r_{\ell}}
$$

where $\mathbf{n}_{r_{\ell}} \sim \mathcal{C N}\left(\mathbf{0}, \mathbf{I}_{N_{R}}\right) \in \mathbb{C}^{N_{R} \times 1}$ denotes the white zeromean circularly symmetric complex Gaussian (ZMCSCG) noise at relay $R_{r_{\ell}}$.

In the second time slot, the selected relay $R_{r_{\ell}}$ processes its received signal $\mathbf{y}_{r_{\ell}}$ by a matrix $\Psi_{r_{\ell}} \in \mathbb{C}^{N_{R} \times N_{R}}$ and then amplifies and forwards it to $D$. The processing matrices satisfy $\operatorname{tr}\left\{\boldsymbol{\Psi}_{r_{\ell}} \boldsymbol{\Psi}_{r_{\ell}}^{H}\right\}=1$. Denote the power amplify factor at relay $R_{r_{\ell}}$ as $w_{\ell}$, and the retransmitted signal is

$$
\mathbf{t}_{r_{\ell}}=w_{\ell} \boldsymbol{\Psi}_{r_{\ell}} \mathbf{y}_{r_{\ell}}=w_{\ell} \boldsymbol{\Psi}_{r_{\ell}} \mathbf{H}_{r_{\ell}} \mathbf{s}+w_{\ell} \boldsymbol{\Psi}_{r_{\ell}} \mathbf{n}_{r_{\ell}} .
$$

Let $P_{R}$ denote the transmit power at each relay (suppose that each relay has the same power constraint),

$$
\begin{aligned}
\mathbb{E}\left\{\operatorname{tr}\left(\mathbf{t}_{r_{\ell}} \mathbf{t}_{r_{\ell}}^{H}\right)\right\} & =w_{\ell}^{2} E\left\{\operatorname{tr}\left(\sigma_{s}^{2} \mathbf{\Psi}_{r_{\ell}} \mathbf{H}_{r_{\ell}} \mathbf{H}_{r_{\ell}}^{H} \mathbf{\Psi}_{r_{\ell}}^{H}+\mathbf{\Psi}_{r_{\ell}} \mathbf{\Psi}_{r_{\ell}}^{H}\right)\right\} \\
& =w_{\ell}^{2} E\left\{\operatorname{tr}\left(\sigma_{s}^{2} \mathbf{H}_{r_{\ell}} \mathbf{H}_{r_{\ell}}^{H} \mathbf{\Psi}_{r_{\ell}}^{H} \mathbf{\Psi}_{r_{\ell}}+\mathbf{\Psi}_{r_{\ell}} \mathbf{\Psi}_{r_{\ell}}^{H}\right)\right\} \\
& =w_{\ell}^{2} \operatorname{tr}\left(\mathbb{E}\left\{\sigma_{s}^{2} \mathbf{H}_{r_{\ell}} \mathbf{H}_{r_{\ell}}^{H} \mathbf{\Psi}_{r_{\ell}}^{H} \mathbf{\Psi}_{r_{\ell}}+\mathbf{\Psi}_{r_{\ell}} \mathbf{\Psi}_{r_{\ell}}^{H}\right\}\right) \\
& =w_{\ell}^{2}\left(\sigma_{s}^{2} \operatorname{tr}\left(\mathbb{E}\left\{\mathbf{H}_{r_{\ell}} \mathbf{H}_{r_{\ell}}^{H} \mathbf{\Psi}_{r_{\ell}}^{H} \mathbf{\Psi}_{r_{\ell}}\right\}\right)+1\right) \\
& \leq P_{R} .
\end{aligned}
$$

The signal received by $D$ is given by

$$
\mathbf{z}=\sum_{\ell=1}^{L} \mathbf{G}_{r_{\ell}} \mathbf{t}_{r_{\ell}}+\mathbf{n}_{D}
$$

where $\mathbf{n}_{D} \sim \mathcal{C N}\left(\mathbf{0}, \mathbf{I}_{N_{D}}\right) \in \mathbb{C}^{N_{D} \times 1}$ denotes the ZMCSCG noise at $D$.

\section{Proposed Eigenmode Selection Algorithm}

In this paper, we use eigenmode construction method [8] for selecting the opportunistic channels. By applying processing matrices at the relay node, $\mathbf{H}_{k}$ and $\mathbf{G}_{k}$ can be degraded to a single eigenmode vector respectively. In other words, relay $R_{k}$ has $N_{R}$ backward eigenmodes associated with $\mathbf{H}_{k}$ and $N_{R}$ forward eigenmodes with $\mathbf{G}_{k}$, and we choose one pair of eigenmodes (one backward mode and one forward mode) for one relay node to cooperate in the transmission, so that the multi-antenna relay node can work as single-antenna relay. Totally, we select $L$ relay nodes, e.g., $L$ eigenmode pairs instead of antenna pairs in [9], to generate equivalent compound backward channel $\mathbf{H}$ and forward channel $\mathbf{G}$.

Let the singular value decomposition (SVD) of relay $R_{k}$ 's backward and forward channel be:

$$
\begin{array}{r}
\mathbf{H}_{k}=\mathbf{U}_{H_{k}} \Lambda_{H_{k}}^{\frac{1}{2}} \mathbf{V}_{H_{k}}^{H}, \\
\mathbf{G}_{k}=\mathbf{U}_{G_{k}} \Lambda_{G_{k}}^{\frac{1}{2}} \mathbf{V}_{G_{k}}^{H}
\end{array}
$$

where $\Lambda_{H_{k}}^{\frac{1}{2}}=\left[\operatorname{diag}\left(\lambda_{H_{k}}(1), \ldots, \lambda_{H_{k}}\left(N_{R}\right)\right), \mathbf{0}^{N_{R} \times\left(N_{S}-N_{R}\right)}\right]$ and $\Lambda_{G_{k}}^{\frac{1}{2}}=\left[\operatorname{diag}\left(\lambda_{G_{k}}(1), \ldots, \lambda_{G_{k}}\left(N_{R}\right)\right), \mathbf{0}^{N_{R} \times\left(N_{D}-N_{R}\right)}\right]^{T}$ are matrices comprising the eigenvalues of $\mathbf{H}_{k}$ and $\mathbf{G}_{k}$ respectively, and

$$
\begin{aligned}
\mathbf{U}_{H_{k}} & =\left[\mathbf{u}_{H_{k}}(1), \ldots, \mathbf{u}_{H_{k}}\left(N_{R}\right)\right] \in \mathbb{C}^{N_{R} \times N_{R}}, \\
\mathbf{U}_{G_{k}} & =\left[\mathbf{u}_{G_{k}}(1), \ldots, \mathbf{u}_{G_{k}}\left(N_{D}\right)\right] \in \mathbb{C}^{N_{D} \times N_{D}}, \\
\mathbf{V}_{H_{k}} & =\left[\mathbf{v}_{H_{k}}(1), \ldots, \mathbf{v}_{H_{k}}\left(N_{S}\right)\right] \in \mathbb{C}^{N_{S} \times N_{S}}, \\
\mathbf{V}_{G_{k}} & =\left[\mathbf{v}_{G_{k}}(1), \ldots, \mathbf{v}_{G_{k}}\left(N_{R}\right)\right] \in \mathbb{C}^{N_{R} \times N_{R}}
\end{aligned}
$$

are unitary matrices of eigenvectors. Then the equivalent compound backward and forward channel are:

$$
\begin{aligned}
\mathbf{H} & =\left[\sqrt{\lambda_{H_{r_{1}}}\left(b_{1}\right)} \mathbf{v}_{H_{r_{1}}}\left(b_{1}\right), \ldots, \sqrt{\lambda_{H_{r_{L}}}\left(b_{L}\right)} \mathbf{v}_{H_{r_{L}}}\left(b_{L}\right)\right]^{H} \\
& \triangleq\left[\mathbf{h}_{b_{1}, r_{1}}, \ldots, \mathbf{h}_{b_{L}, r_{L}}\right]^{H}, \\
\mathbf{G} & =\left[\sqrt{\lambda_{G_{r_{1}}}\left(f_{1}\right)} \mathbf{u}_{G_{r_{1}}}\left(f_{1}\right), \ldots, \sqrt{\lambda_{G_{r_{L}}}\left(f_{L}\right)} \mathbf{u}_{G_{r_{L}}}\left(f_{L}\right)\right] \\
& \triangleq\left[\mathbf{g}_{f_{1}, r_{1}}, \ldots, \mathbf{g}_{f_{L}, r_{L}}\right]
\end{aligned}
$$

where $b_{\ell}$ and $f_{\ell}(\ell=1, \ldots, L)$ denote the selected mode of backward channel matrix and forward channel matrix of relay $R_{r_{\ell}}$ respectively.

Due to the processing matrices, the non-selected modes do not work in the transmission. Let the received signals at the $L$ selected backward channels be stacked into a vector $\mathbf{y}$, and $\mathbf{n}_{R}$ is the corresponding noise,

$$
\mathbf{y}=\mathbf{H s}+\mathbf{n}_{R} .
$$


Let diagonal matrix $\mathbf{W}=\operatorname{diag}\left(w_{1}, \ldots w_{L}\right)$ represent the $\mathrm{AF}$ relay amplifying function. The signal retransmitted can be written as $\mathbf{t}$

$$
\mathbf{t}=\mathbf{W} \mathbf{y}=\mathbf{W H s}+\mathbf{W} \mathbf{n}_{R} .
$$

Then (5) can be rewritten as

$$
\mathbf{z}=\mathbf{G t}+\mathbf{n}_{D}=\mathbf{G W H}+\mathbf{G W} \mathbf{n}_{R}+\mathbf{n}_{D} .
$$

Furthermore, by denoting $\mathbf{H}_{e q}=\mathbf{G W H}$ and $\mathbf{n}_{e q}=$ $\mathbf{G W} \mathbf{n}_{R}+\mathbf{n}_{D}$, (13) can be reduced to $\mathbf{z}=\mathbf{H}_{e q} \mathbf{s}+\mathbf{n}_{D}$. According to [10], the MSE of symbol estimation will achieve the minimum when Wiener filter is used. The corresponding MSE is

$$
\begin{aligned}
Q & =\sigma_{s}^{2} \operatorname{tr}\left\{\left(\mathbf{I}_{N_{D}}+\sigma_{s}^{2} \mathbf{H}_{e q} \mathbf{H}_{e q}^{H} \boldsymbol{\Phi}^{-1}\right)^{-1}\right\}+\sigma_{s}^{2}\left(N_{S}-N_{D}\right) \\
& \triangleq \sigma_{s}^{2} \operatorname{tr}\left\{\boldsymbol{\Phi}\left(\boldsymbol{\Phi}+\sigma_{s}^{2} \mathbf{H}_{e q} \mathbf{H}_{e q}^{H}\right)^{-1}\right\}+\beta,
\end{aligned}
$$

where $\boldsymbol{\Phi}=\mathbb{E}\left\{\mathbf{n}_{e q} \mathbf{n}_{e q}^{H}\right\}=\mathbf{G W}(\mathbf{G W})^{H}+\mathbf{I}_{N_{D}}$ and $\beta$ is a constant, which will be omited hereafter.

From (14), we can use a brute-force search to minimize $Q$ and obtain the best relay set. However, such greedy search is impractical, since the required times of trials will be enormously increased as the number of antennas and relays increases. In [9], a suboptimal search method is proposed, which can optimize the system asymptotically.

Let $(m, n, k)$ denote the $m$ th backward eigenmode and $n$th forward eigenmode of $k$ th relay. $\mathbf{H}_{\ell}=\left[\mathbf{h}_{b_{1}, r_{1}}, \ldots, \mathbf{h}_{b_{\ell}, r_{\ell}}\right]^{H}$ and $\mathbf{G}_{\ell}=\left[\mathbf{g}_{f_{1}, r_{1}}, \ldots, \mathbf{g}_{f_{\ell}, r_{\ell}}\right]$ are the already selected backward and forward channel in the $\ell$ th iteration respectively. $\mathbf{W}_{\ell}$ is the corresponding AF function. Assume full power in (4), and relay gain $w_{m, k}$ associated with $\mathbf{h}_{m, k}$ can be calculated as

$$
w_{m, k}=\sqrt{P_{R} /\left(\sigma_{s}^{2}\left|\mathbf{h}_{m, k}\right|^{2}+1\right)} .
$$

Denote

$$
\begin{aligned}
& \mathbf{\Phi}_{\ell} \quad=\quad \mathbf{I}_{N_{D}}+\mathbf{G}_{\ell} \mathbf{W}_{\ell}^{2} \mathbf{G}_{\ell}^{H}, \\
& \mathbf{A}_{\ell}=\boldsymbol{\Phi}_{\ell}+\sigma_{s}^{2}\left(\mathbf{G}_{\ell} \mathbf{W}_{\ell} \mathbf{H}_{\ell}\right)\left(\mathbf{G}_{\ell} \mathbf{W}_{\ell} \mathbf{H}_{\ell}\right)^{H}, \\
& \mathbf{F}_{m, n, k}=\mathbf{G}_{\ell} \mathbf{W}_{\ell} \mathbf{H}_{\ell}+w_{m, k} \mathbf{g}_{n, k} \mathbf{h}_{m, k}^{H}, \\
& \mathbf{p}_{m, n, k}=\sigma_{s}^{2}\left(\mathbf{G}_{\ell} \mathbf{W}_{\ell} \mathbf{H}_{\ell}\right) w_{m, k} \mathbf{h}_{m, k}+w_{m, k}^{2} \mathbf{g}_{n, k}, \\
& \mathbf{q}_{m, n, k}=\sigma_{s}^{2} \mathbf{F}_{m, n, k} w_{m, k} \mathbf{h}_{m, k}, \\
& \mathbf{B}_{(m, n, k)}^{-1}=\quad \mathbf{A}_{\ell}^{-1}-\frac{\mathbf{A}_{l}^{-1} \mathbf{p}_{m, n, k} \mathbf{g}_{n, k}^{H} \mathbf{A}_{\ell}^{-1}}{1+\mathbf{g}_{n, k}^{H} \mathbf{A}_{\ell}^{-1} \mathbf{p}_{m, n, k}}, \\
& \mathbf{C}_{(m, n, k)}^{-1}=\mathbf{B}_{(m, n, k)}^{-1}-\frac{\mathbf{B}_{(m, n, k)}^{-1} \mathbf{g}_{n, k} \mathbf{q}_{m, n, k}^{H} \mathbf{B}_{(m, n, k)}^{-1}}{1+\mathbf{q}_{m, n, k}^{H} \mathbf{B}_{(m, n, k)}^{-1} \mathbf{g}_{n, k}} .
\end{aligned}
$$

Proposition [9]: The MSE resulted from the $(\ell+1)$ th additional eigenmode $(m, n, k)$ is

$$
Q_{\ell+1}^{(m, n, k)}=\sigma_{s}^{2} \operatorname{tr}\left\{\left(\mathbf{\Phi}_{\ell}+w_{m, k} \mathbf{g}_{n, k} \mathbf{g}_{n, k}^{H}\right) \mathbf{C}_{(m, n, k)}^{-1}\right\} .
$$

By using (17), an MMSE based relay selection algorithm is proposed as shown in Algorithm 1.

Meanwhile, we obtain the processing matrices at $R_{r_{\ell}}(\ell=$ $1, \ldots, L)$ for the selected mode $\left(b_{\ell}, f_{\ell}\right)$ :

$$
\boldsymbol{\Psi}_{r_{\ell}}=\mathbf{v}_{G_{r_{\ell}}}\left(f_{\ell}\right) \mathbf{u}_{H_{r_{\ell}}}^{H}\left(b_{\ell}\right) .
$$

Algorithm 1 MMSE based greedy eigenmode selection for MPA relay system

1) Initialization: Set $\ell=0, \mathbf{H}_{\ell}=\mathbf{G}_{\ell}=\mathbf{W}_{\ell}=\emptyset, \mathbf{A}_{\ell}=$ $\boldsymbol{\Phi}_{\ell}=\mathbf{I}_{N_{D}}$, previous_MSE $=+\infty$; We define a set of eigenmodes decribed by $\Omega$

$$
\Omega=\left\{(m, n, k) \mid \lambda_{H_{k}}(m) \geq \lambda_{0}, \lambda_{G_{k}}(n) \geq \lambda_{0}\right\}
$$

where $\lambda_{0}$ is a threshold value (we will discuss the value in Section IV), which is used to limit the search domain. According to (18), the candidate eigenmode pair set can be denoted by

$$
\Gamma=\left\{(m, n, k) \in \Omega \mid\left(\mathbf{h}_{m, k}, \mathbf{g}_{n, k}\right)\right\} .
$$

2) Iterations: Compute $\mathbf{W}_{\ell}, \boldsymbol{\Phi}_{\ell}$ and $\mathbf{A}_{\ell}$, for each eigenmode pair $\left(\mathbf{h}_{m, k}, \mathbf{g}_{n, k}\right)$ in $\Gamma$, calculate $Q_{\ell+1}^{(m, n, k)}$, and select the $(\ell+1)$ th eigenmode pair by:

$$
\left(b_{j}, f_{j}, r_{j}\right)_{j=\ell+1}=\underset{(m, n, k) \in \Omega}{\arg \min }\left\{Q_{\ell+1}^{(m, n, k)}\right\} .
$$

3) If $\min \left\{Q_{\ell+1}^{(m, n, k)}\right\}<$ previous_MSE, then previous_MSE $=\min \left\{Q_{\ell+1}^{(m, n, k)}\right\}$. Update:

$$
\begin{aligned}
& \Omega=\Omega-\left\{\left(b_{\ell+1}, f_{\ell+1}, r_{\ell+1}\right)\right\} ; \\
& \mathbf{H}_{\ell+1}=\left[\mathbf{H}_{\ell}^{H}, \mathbf{h}_{b_{\ell+1}, r_{\ell+1}}\right]^{H}, \\
& \mathbf{G}_{\ell+1}=\left[\mathbf{G}_{\ell}, \mathbf{g}_{f_{\ell+1}, r_{\ell+1}}\right], \\
& \mathbf{W}_{\ell+1}=\operatorname{diag}\left(\mathbf{W}_{\ell}, w_{b_{\ell+1}, r_{\ell+1}}\right) ; \\
& \ell=\ell+1 ;
\end{aligned}
$$

Go to step 2.

Else, end up the iteration and set $\mathbf{H}_{\ell}$ and $\mathbf{G}_{\ell}$ as the selected channels.

These processing matrices are used to null out all non-selected backward and forward eigenmodes at relay $R_{r_{\ell}}$.

To see it from another perspective, suppose that each relay receives the signal from $S$ with a received beamforming matrix $\mathbf{W}_{S R_{k}}=\mathbf{U}_{H_{k}}^{H}$ and retransmits the signal with beamforming matrix $\mathbf{W}_{R_{k} D}=\mathbf{V}_{G_{k}}$. By doing so, the backward channel $\mathbf{H}_{k}$ and forward channel $\mathbf{G}_{k}$ can be rewritten as their equivalent form similar to (9) and (10). Then we select the antenna pairs using the method in [9]. The result will be just the same as our proposed scheme. Furthermore, $\mathbf{W}_{S R_{k}}$ and $\mathbf{W}_{R_{k} D}$ are unitary matrices, which will not bring any unfairness on power compared to [9]. That is to say, our scheme is feasible and comparable, and the simulation in the next section will demonstrate that it is competent.

\section{Simulation Results}

In our simulation experiment, we use Monte-Carlo method to test our proposed scheme. All the channels involved are modeled as i.i.d ZMCSCG random variables with unit covariance. In our simulations, we setup a realistic MIMO relay system as $N_{S}=N_{D}=4, N_{R}=2$. The power constraint at relay nodes $P_{R}$ is $5 d B$ above the noise in our simulation.

[11] demonstrates that the sum rate of a MIMO broadcast 
channel can be achieved by dirty paper coding (DPC). According to [8], the best capacity scaling we may achieve is that of a DPC approach, since we are applying a MIMO multiuser technique. Therefore, we heuristically set the threshold $\lambda_{0}=\log K$ in our simulation. This means that we will set a higher threshold to select the eigenmodes as the number of relay nodes increases. What is more, it should be pointed out that this is not an optimal threshold, but is feasible and practical.

In [7] and [8], the proposed schemes both select $M$ relays/antennas to achieve full multiplexing gain. [9] uses Greedy MSE Minimization algorithm for SPA relay. Fig. 2 compares the frequency histogram of the selected relays for SPA and MPA relay system at $P_{S}=0 d B$ and $K=40$. We can see that in our MPA setup, the number of turned on relays is smaller than the one of SPA. That means we cost less overhead than the existing scheme.

Fig. 3 shows the MSE performance versus $P_{S}$ for SPA and MPA setup. The MSE curve of our scheme is lower than that of SPA in the two cases $(K=8$ and $K=15)$. Combining it with Fig. 2, we will see that the proposed scheme cost less overhead but obtain better performance in terms of MSE.

Fig. 4 illustrates how the MSE performance reflects in BER. We set 11 simulation points as $P_{S}=0,2, \ldots, 20 d B$, and each point is simulated by 50000 transmissions. In our simulation, we set the transmit power at each relay as $N_{S} P_{R} / L$, to make the two configurations be fair on power allocation. Similar to Fig. 3, obviously our scheme outperforms the SPA setup. The gain gap between SPA and MPA becomes larger when the number of candidate relays increases, which implies that with a larger number of candidate relays, greater advantages in the performance of our strategy is bound to appear.
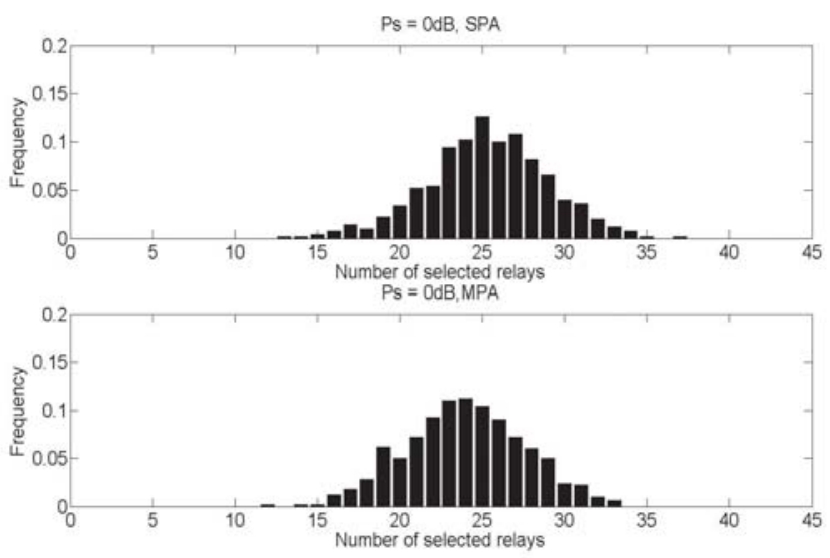

Fig. 2. Frequency histogram of relays.

\section{CONCLUSION}

In this paper, we propose an MMSE based eigenmode selection algorithm for an AF MIMO relay system, by selecting an additional relay eigenmode to minimize the MSE iteratively. We consider the relay nodes with multi-antenna configuration,

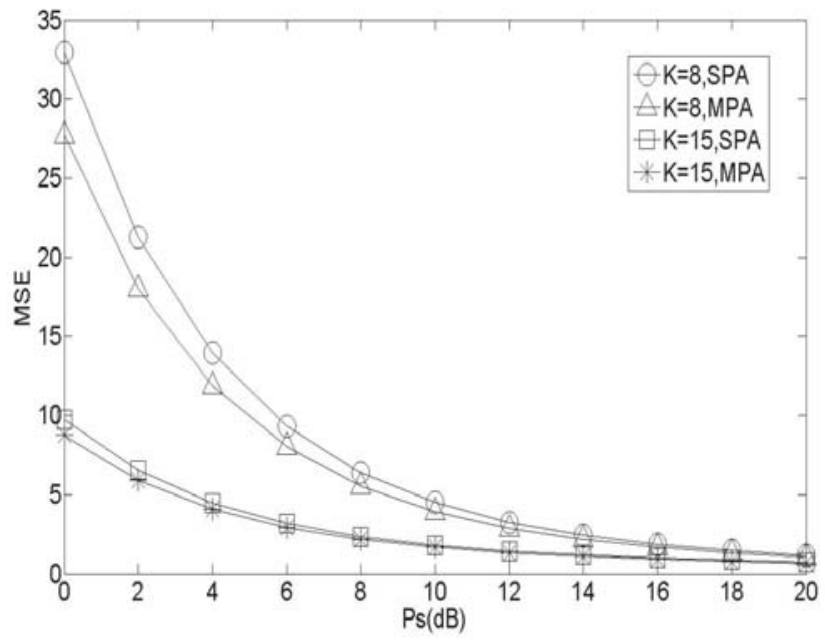

Fig. 3. MSE performance comparison.

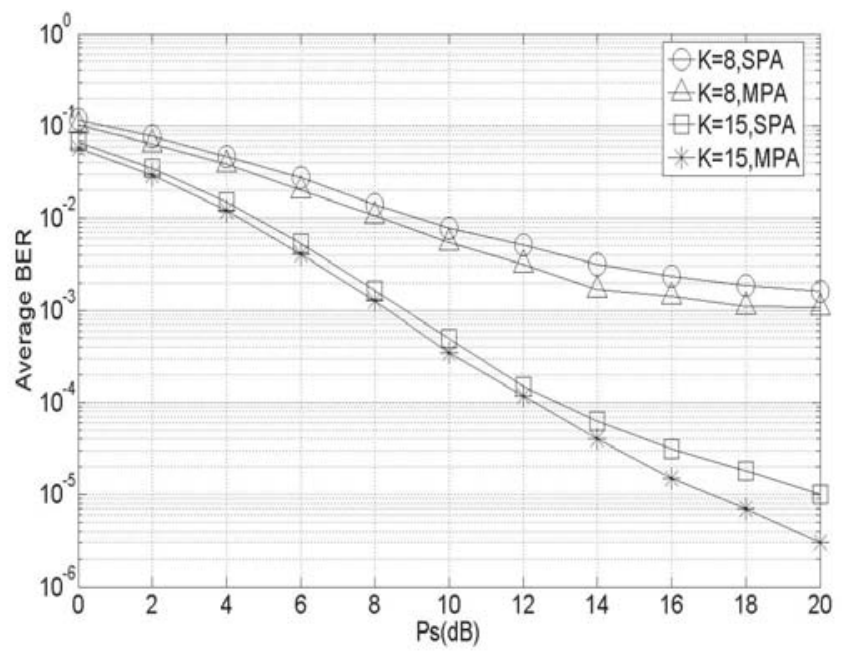

Fig. 4. BER performance comparison.

which is more practical than SPA setup. Simulation results show that the proposed algorithm outperforms the existing one in terms of MSE and BER.

\section{ACKNOWLEDGEMENT}

This work is supported by the National 973 Project \#2012CB316106, by NSF China \#60972031 and \#61161130529, by the National 973 Project \#2009CB824904.

\section{REFERENCES}

[1] A. Bletsas, A. Khisi, D. F. Reed, and A. Lippman, "A simple cooperative diversity method based on network path selection", IEEE J. Select. Areas. Commun., vol. 24, no. 3, pp. 659-672, Mar. 2006.

[2] Yindi Jing, and H. Jafarkhani, "Single and multiple relay selection schemes and their achievable diversity orders", IEEE Trans. on Wireless Comm., vol. 8, no. 3, Mar. 2009. 
[3] A. Bletsas, A. Lippman, and D. F. Reed, "A simple distributed method for relay selection in cooperative diversity wireless networks, based on reciprocity and channel measurements", Vehicular Technology Conference, 2005. VTC 2005-Spring. 2005 IEEE 61st.

[4] H. Adam, C. Bettstetter, and S. M. Senoucim, "Adaptive relay selection in cooperative wireless networks", Proc. IEEE Symposium on PIMRC, Cannes, France, Sep.15-18, 2008.

[5] B. Wang, J. Zhang, and A. Host-madsen, "On the capacity of MIMO relay channels", IEEE Trans. Inf. Theory, vol. 51, pp. 29-43, Jan. 2005.

[6] A. Wittneben, and B. Rankov, "Distributed antenna systems and linear relaying for gigabit MIMO wireless", in Proc. IEEE Vehicular Technology Conference, VTC Fall, Los Angeles, USA, Sep. 2004.

[7] W. Zhang and K. B. Letaief, "Opportunistic relaying for dual-hop wireless MIMO channels", in Proc. IEEE Global Telecom. Conf., New Orleans, LA, pp. 3985-3989, Nov. 2008.

[8] M. A. Torabi and J. F. Frigon, "Semi-orthogonal relay selection and beamforming for amplify-and-forward MIMO relay channels", in Proc. IEEE Wireless Communications and Networking Conf., Las Vegas, NV, Mar. 2008, pp. 48-53.

[9] M. Ding, S. Liu, H. W. Luo, and W. Chen, "MMSE based greedy antenna selection scheme for AF MIMO relay systems", IEEE Signal. Proc. Lett. , vol. 17, No. 5, May. 2010.

[10] W. Guan, and H. W. Luo, "Joint MMSE transceiver design in nonregenarative MIMO relay systems", IEEE Commun. Lett., vol. 12, no. 7, pp. 517-519, Jul. 2008

[11] M. Sharif and B.Hassabi, "A comparison of time-sharing, DPC and beamforming for MIMO broadcast channels with many users" IEEE Trans. Commun., vol. 55, no. 1, pp. 11-15, Jan. 2007. 